\title{
A Novel Traffic Control Scheme for Multi- Layered Satellite Network Based on Chain Reaction Model
}

\author{
http://dx.doi.org/10.3991/ijoe.v12i01.5167 \\ Ning Li, Pengfei Wang, Zhongliang Deng, Ke Han \\ Beijing University of Posts and Telecommunications. Beijing, China
}

\begin{abstract}
Multi-Layered Satellite Network (MLSN) is a kind of delay tolerant network with characters of intermittent connection, long packet queuing time, complex and uncertain delay time, etc. The call congestion rate of new business is higher in delay tolerant network which leads to the reduction of communication quality and the high packet loss rate. The existing traffic control scheme is hard to meet the individual requirements of new business. It becomes more and more important to study the most effective traffic control scheme so as to increase the satellite bandwidth utilization. According to the nuclear chain reaction of atomic physics, a novel traffic control method based on chain reaction model (TCCR) is presented in this paper to realize dynamic data transmission and control for MLSN. The optimal flow distribution scheme will be obtained by analyzing the network transmission information in this algorithm. Simulation by OPENT is done which proves that TCCR can obviously improve the network total throughput, shorten link transmission delay, and reduce packet loss rate of MLSN.
\end{abstract}

Index Terms-GEO, LEO, Chain Reaction, Traffic Control, Satellite Communications.

\section{INTRODUCTION}

With the development of the wireless communication technology, the demand for broadband communication services has increased rapidly. Satellite communications network plays an important role not only by providing broadband access directly, but also being part of the worldwide core network [1]. MLSN is a kind of delay tolerant network with characters of intermittent connection, long packet queuing time, complex and uncertain delay time, etc. The call congestion rate of new business is higher in delay tolerant network which leads to the reduction of communication quality and the high error code rate. According to statistics, when the bitrate of LEO satellite reaches to $350 \mathrm{Kbps}$, the packet drop rate will be more than $30 \%$, and the transmission delay will be $300 \mathrm{~ms}$. The existing traffic control scheme is hard to meet new the individual requirements of new business. In order to guarantee satellite network resources reasonable use, it becomes more and more important to study the most effective traffic control scheme so as to increase the satellite bandwidth utilization.

For traffic control, many researchers have proposed a variety of effective schemes. Hiroki proposes the load balancing and QoS provisioning based on congestion prediction (LBQP) for GEO/LEO Hybrid satellite network to improve the efficiency of the GEO/LEO hybrid satellite networks and the QoS satisfaction of end users [2]. But the parameter and threshold selection of LBQP is too simple which would lead to all sorts of problems. Cen proposes a dynamic coverage adjustment scheme that changes beam size and resource allocation according to the spatial distribution of user traffic [3]. More resource is offered to the beam covering the hot spot, whereas basic coverage is maintained for the surrounding area. However, to make this model's results satisfied, it needs a lot of traffic data which led to a low efficiency of the traffic control. Kawamoto designs a delay-based traffic distribution technique for MLSN [4]. This method focus on traffic control in two-layered networks, especially on distributing the packet flow between the two layers in order to minimize the transfer delay of the network. Nevertheless, topologic structure of this model is too complicated so it is very difficult to be used widely in practice due to its high complexity.

In order to handle the shortage existing in the previous traffic control scheme, a novel traffic control method based on chain reaction model (TCCR) is presented in this paper to realize dynamic data transmission and control for MLSN. The optimal flow distribution scheme will be given by analyzing the network transmission information in this algorithm. Simulation by OPENT is done which proves that TCCR can obviously improve the network total throughput, shorten link transmission delay, and reduce packet loss rate of MLSN.

\section{SYSTEM MODEL}

Non-Geostationary Earth Orbit (NGEO) satellite networks have gained research attention. Since they offer many features, e.g., extensive coverage, disasterresistance, and efficient power consumption, they are considered as a good candidate for providing global communication services [5]. MLSN, which consist of layered NGEO satellite networks, have attracted much attention since they achieve excellent load distribution through by passing traffic from the lower layer to upper layer [6]. In this paper, we focus on traffic control in two-layered networks composing of LEO and GEO satellites, and the system configuration is shown in Fig. 1. In MLSN, there is a strong correlation in traffic data between LEO-LEO and LEO-GEO. As shown in Fig.1, the satellites in MLSN will affect each other in the process of data forwarding according to the principle of flow conservation [7]. However, most previous researches only show their concern to single satellite in traffic assignment and control, while the study with regard to the interaction between satellites is 
rare. Therefore, the original methods cannot meet the needs of the current satellite system, resulting in decreased system efficiency. In order to handle the problem, this thesis pays emphasis on analyzing the correlation between different satellites in MLSN and the Chain Reaction Model of satellite traffic transmission is presented by analogy with the nuclear chain reaction.

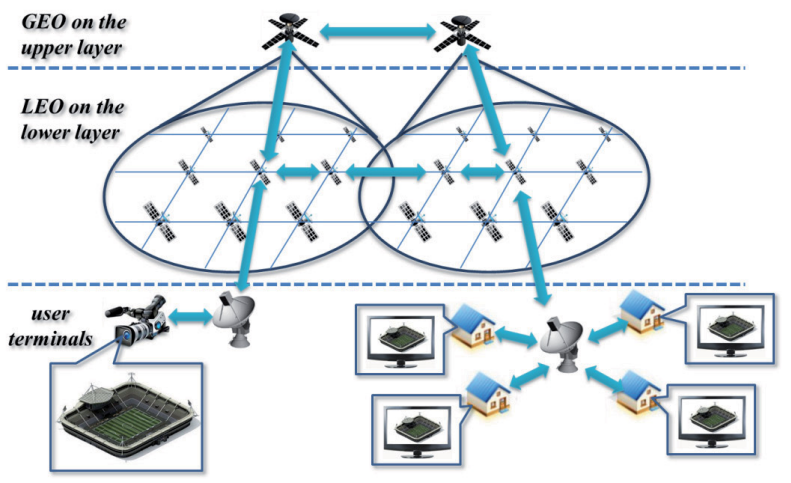

Figure 1. The system model

\section{A. The Chain Reaction Model in MLSN}

The core of chain reaction is the construction of "chain" and the transmission of "free radicals" [8]. In MLSN, "chain" refers to the inter satellite links (ISL), and the traffic data of ISL can be seen as "free radicals". As shown in Fig.1, the basic component of MLSN is composed of one GEO and all the LEOs within the coverage area of the GEO. The specific architecture of this unit is shown in figure 2 - (1), where A, B, C, D, X represents the LEO on the lower layer, and G represents the GEO on the upper layer.

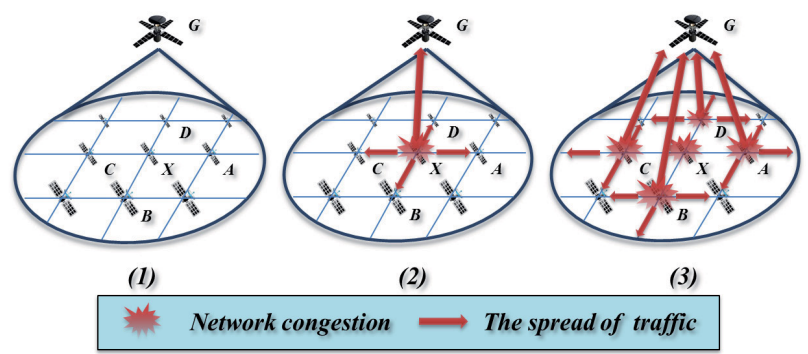

Figure 2. Chain reaction in satellite network

The chain reaction of MLSN is shown in Figure2-(1), (2), (3). In 2-(1), when one of the LEOs within the coverage area of the GEO, for example X, enters overload condition due to the occurrence of network congestion, $\mathrm{X}$ will send warning notices to the GEO and satellites A, B, C, D in order to reduce the input flow of its neighboring satellites. After receiving the warning notice, A will enter overload condition because of the reduction of data traffic could be carried by link A-X and A-G. Then A will send warning notices to its neighboring satellites to reduce the input flow. What happened in B, C and D is the same with A. The spread of warning notices result in sharp increases in overloaded satellite of MLSN and this process could be defined as the Chain Reaction in MLSN which is shown in Fig.2-(3).

The formation and propagation of "free radicals" plays an important role to the process and scale of chain reaction directly which has the same characteristics with the transmission of traffic data in MLSN. In MLSN, the varia- tion of "free radicals" is affected by two aspects: traffic data coming from neighboring LEO satellites $S$ and user terminals within the coverage area $L_{i}$. The latter accounts for a far higher proportion of total input traffic data. Moreover, there is a negative correlation between two neighboring satellites connected by the same "chain" in MLSN. For link X-A in Fig.2, the output flow of X $\left(X_{o}\right)$ is approximately equal to the input flow of $\mathrm{A}\left({ }^{A_{i}}\right)$ under ideal conditions. $X_{o}$ is always keep pace with changes in $A_{i}$. Therefore, the core of constructing the Chain Reaction Model in MLSN is to carefully research on fullcorrelation features of related satellites.

\section{B. The key parameters}

\section{1) The discharge coefficient $(U)$}

The discharge coefficient $U$ is one of the important parameters to describe the lower layer satellite traffic fluctuation. According to the Law of Conservation of Energy in atomic physics, $U$ obeys the flow conservation of satellite system at first which means that in a certain time period, the output flow of satellite is approximately equal to the input flow of satellite. As shown in Fig.3, the input flow in the previous time slot of LEO's neighboring satellites $A, B, C$ are denoted by $A_{i}, B_{i}, C_{i}$, respectively, and the input flow in current time slot are denoted by $A_{i}^{\prime}, B_{i}^{\prime}, C_{i}^{\prime}$. Then the sum of input flow of LEO's neighboring satellites $L_{i}$ can be expressed as:

$$
L_{i}=A_{i}+B_{i}+C_{i}
$$

According to the negative correlation between two neighboring satellites connected by the same "chain" in MLSN, we can get:

$$
\begin{gathered}
\Delta S=S^{\prime}-S=-\Delta L_{i} \\
\Delta A_{i}=\Delta L_{i} \times \frac{A_{i}}{L_{i}} \\
A_{i}^{\prime}=A_{i}+\Delta A_{i} \\
A_{i}^{\prime}=A_{i}+\left(1+\frac{\Delta L_{i}}{L_{i}}\right)
\end{gathered}
$$

Where $\Delta S$ refers to the satellite traffic variations coming from user terminals within the coverage area of LEO, $\Delta L$ refers to the satellite traffic variations coming from neighboring LEO satellites. Therefore, $U$ can be expressed as follows:

$$
U=\frac{A_{i}^{\prime}}{A_{i}}=1+\frac{\Delta L_{i}}{L_{i}}=1-\frac{S^{\prime}-S}{F_{i}-S}
$$

Where $S$ and $S^{\prime}$ refers to the satellite traffic coming from user terminals within the coverage area of LEO in the previous and current time slot, respectively. $F_{i}$ refers 
to the sum of input flow of LEO which can be expressed as follows:

$$
F_{i}=A_{i}+B_{i}+C_{i}+S
$$

The value $U$ of is negative on account of the confinement $S^{\prime}<F_{i}$ and there are three situations as follows:

- $U>1$ : means the traffic flow of the link is declining;

- $1>U>0$ : means the traffic flow of the link is increasing;

- $U=1$ : means the traffic flow of the link remains unchanged.

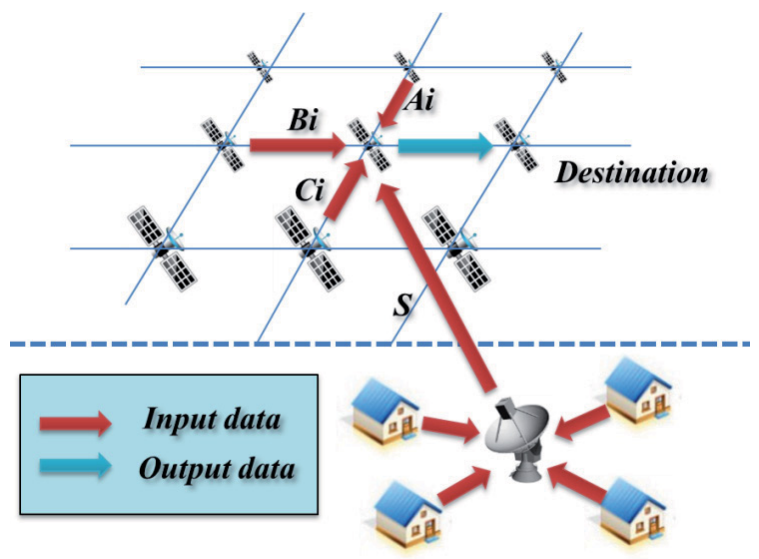

Figure 3. Input data and output data in LEO network

In MLSN, the discharge coefficient needs to be modified because of the multilayer network architecture. As shown in Fig.4, the input flow in the previous time slot of LEOs within the coverage area of GEO are denoted by $A_{g}, B_{g}, \mathrm{~K}, N_{g}$, respectively, and then the sum of input flow of GEO can be expressed as:

$$
F_{g}=A_{g}+B_{g}+\mathrm{L}+N_{g}+S_{g}
$$

The correction of discharge coefficient $U_{g}$ can be obtained by:

$$
U_{g}=1-\frac{S_{g}^{\prime}-S_{g}}{F_{g}-S_{g}}
$$

Where $S_{g}$ refers to the upper layer satellite traffic.

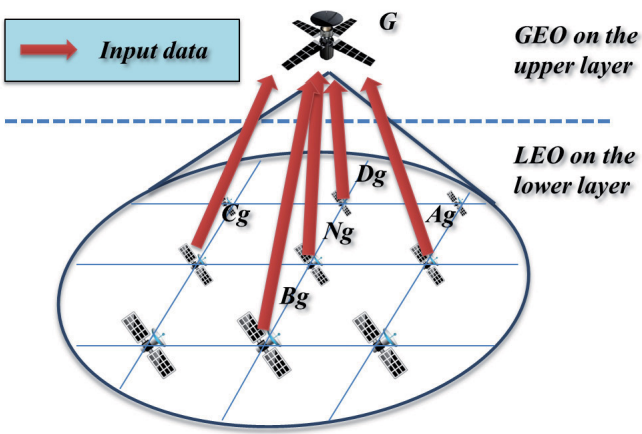

Figure 4. Input data in MLSNs

\section{2) The attenuation factor}

The attenuation factor $K$ is an important parameter to describe the correlation features of related satellites. In nuclear physics, a quantity is subject to exponential decay if it decreases at a rate proportional to its current value. Symbolically, this process can be expressed by the following differential equation, where $\mathrm{N}$ is the quantity and $\lambda$ is a positive rate called the exponential decay constant:

$$
\lambda=\frac{d N / d t}{N(t)}
$$

A more intuitive characteristic of exponential decay for many people is the time required for the decaying quantity to fall to one half of its initial value. This time is called the half-life, and often denoted by the symbol $t 1 / 2$. The halflife can be written in terms of the decay constant, or the mean lifetime, as:

$$
t_{1 / 2}=\frac{\ln 2}{\lambda}
$$

The concept of decay constant and half-life is introduced into MLSN, and the attenuation factor can be obtained by following the steps below:

step 1. According to the conversion relations of halflife and decay constant, choose natural logarithm as the function model of the attenuation factor;

step 2. By analyzing the characteristic of natural logarithm function, choose logarithmic function to replace.

step 3. Combine the satellite link characteristic parameters with the half-life conversion function, and introduce the control parameters;

step 4. Determine the value range of the parameters and finish the correction of $K$.

Through the above steps, the definition of $K$ can be obtained by:

$$
K=-Q^{H} \times \log U
$$

Using the above expression of $U$ in Eq. $6, K$ can be represented as follows:

$$
K=-Q^{H} \times \log \left(1-\frac{S^{\prime}-S}{F_{i}-S}\right)
$$

Where $H$ is the Hurst parameter which is used as a measure of the burstiness of satellite network traffic flow [9][10], $Q$ is a constant coefficient which relates to channel bandwidth, PDR and etc. The variation speed of $K$ increases with the decreasing of $U$ and limited by constant coefficient $Q$ at the same time.

In MLSN, all the parameters have been added a subscript $g$ to distinguish with the LEO network. The attenuation factor in MLSN is represented as follows: 


$$
K_{g}=-\sqrt[n]{P^{\sum^{H}}} \times \log U_{g}
$$

Where $P$ is a constant coefficient similar to $Q . n$ refers to the number of LEO within the coverage area of the GEO. $P$ and $Q$ will be discussed in next section further. Using the expression of $U_{g}$ in Eq. 9, $K_{g}$ can be represented as follows:

$$
K_{g}=-\sqrt[n]{P^{\sum^{H}}} \times \log \left(1-\frac{S_{g}^{\prime}-S_{g}}{F_{g}-S_{g}}\right)
$$

\section{3) The constant coefficient $Q$ and $P$}

The value of $Q$ depends on channel bandwidth, PDR and etc. From Eq.12, we can conclude that the variation speed of $K$ increases with the decreasing of $U$ and limited by constant coefficient $Q$ at the same time.

Using the expression of $K$ in Eq. 12, we can get:

$$
Q=-\sqrt[H]{\frac{K}{\log U}}
$$

Through the above analysis, it can be summarize that the control of input flow coming from neighboring LEO satellites is associated with the attenuation factor $K$ and the input flow is determined by $Q$ directly when $U$ and $H$ is a certain value. In order to maximize the system throughput, the input flow coming from neighboring LEO satellites should be as much as possible within the total services carrying capacity of LEO. For each LEO in MLSN, the increasing of $Q$ will increase its own traffic flow and also will increase the traffic flow of other neighboring satellites. Therefore, we need to find a suitable value with the aim of shortening transmission delay while using network resource efficiently.

According to the negative correlation between two neighboring satellites connected by the same "chain" in MLSN, we can get that the total input traffic flow of each LEO should be the minimum value of the system bandwidth $B_{w}$, the capacity of current link $F$ and the forwarding capabilities of neighboring LEO satellites $A_{o}$ :

$$
A_{i}=\min \left(B_{w}, F, A_{o}\right)
$$

When $1>U>0$, introduce Eq.12 into Eq.24 and get:

$$
A_{i}^{\prime}=A_{i} \times \frac{1}{1+K}=A_{i} \times \frac{1}{1-Q^{H} \times \log U}
$$

Through the above analysis, the problem has become how to choose a suitable $Q$ to maximize $A_{i}^{\prime}$ under the condition of Eq.21, 22 and 23. In order to study the relationship between $Q, A_{i}^{\prime}$ and $F$, the change of $Q$ was simulated by fixing the values of other parameters.
Meanwhile, to facilitate the analysis of the results, the value of $A_{i}$ was set to 1 .

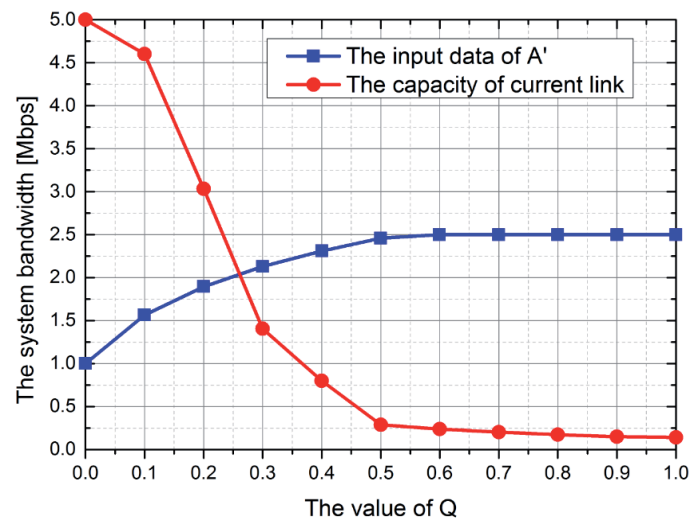

Figure 5. The input data of $\mathrm{A}$ and the capacity of current link versus $\mathrm{Q}$

Fig. 5 is the simulation result of the input data of $A_{i}^{\prime}$ and the capacity of current link $F$ versus Q. As Fig.5 shows, the value of $A_{i}^{\prime}$ will increase step by step with the increase of the $\mathrm{Q}$, but the increase speed will reduce gradually. When the value comes to 0.557 , it remains unchanged due to the limitation of current satellite link bandwidth. Instead, $F$ will decrease with the increase of $\mathrm{Q}$ and the decrease rate is slower in beginning and then becomes faster, at last comes to stable. This is because of the augment of $\mathrm{Q}$ leads to the increasing of input flow from neighboring satellites. The optimal value of $\mathrm{Q}$ which achieves the maximize data forwarding in the LEO network is determined to be 0.258 according to this simulation.

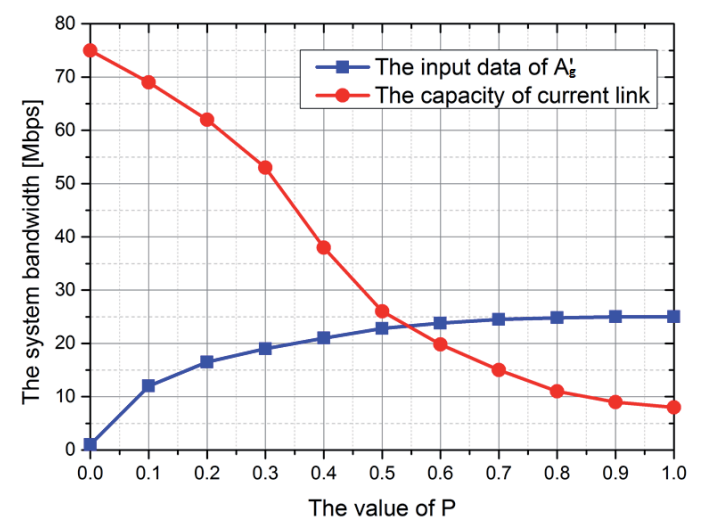

Figure 6. The input data of A and the capacity of current link versus $\mathrm{P}$

Fig.6 is the simulation result of the input data of $A_{g}{ }^{\prime}$ and the capacity of current link $F$ versus P. As Fig.6 shows, the situation of $\mathrm{P}$ is similar to Q. But in GEO network, there is larger bandwidth which makes the LEO satellite network data transmission would not significantly affect the GEO network traffic and the optimal value of $P$ is determined to be 0.546 as a result. 


\section{The Traffic CONTROL Scheme BASED ON CHAIN REACTION MODEL}

Based on above analysis in section 2, the TCCR control scheme can be divided into two stages: LEO network flow control and LEO-GEO integrated network traffic control. The control flow of these two stages is shown as follow:

Stage 1: LEO network flow control

step 1. Based on the satellite traffic variations coming from user terminals within the coverage area of LEO $\Delta S$, calculate the discharge coefficient $U$;

step 2. Initialize the Hurst parameter, constant coefficient $Q$ and current attenuation factor $K$;

step 3. Control current input flow of LEO $T_{i}^{\prime}$ according to Eq.19, where $T_{i}$ refers to input flow in the previous time slot:

$$
T_{i}^{\prime}=\left\{\begin{array}{l}
U \times T_{i}, U \geq 1 \\
T_{i} \times \frac{1}{1+K}, 1>U>0
\end{array}\right.
$$

step 4. As shown in Fig.2-(1), get $\mathrm{K}$ and $\mathrm{U}$ of link $\mathrm{X}$ $A$ and $X-B$, respectively, then calculate the traffic flow from $\mathrm{X}$ to $\mathrm{A} T_{i(A)}{ }^{\prime}$ and $\mathrm{B} T_{i(B)}{ }^{\prime}$ according to Eq. 24 ;

Control output flow of LEO $T_{o}$ as follows:

- $T_{o}<T_{i(A)}{ }^{\prime}$ : means the target satellite node $\mathrm{A}$ is able to forward all the business data coming from the current LEO, and all the traffic flow will be send to A.

- $T_{i(A)}{ }^{\prime}<T_{o}<T_{i(A)}{ }^{\prime}+T_{i(B)}{ }^{\prime}$ : means A cannot forward all the business data coming from the current LEO and the excess of traffic flow needs to be detoured to satellite node B.

- $T_{o}>T_{i(A)}{ }^{\prime}+T_{i(B)}{ }^{\prime}$ : means the neighboring LEO satellites $\mathrm{A}$ and $\mathrm{B}$ cannot forward all the business data both, and the excess of traffic flow needs to be detoured to the GEO on the upper layer.

\section{Stage 2: LEO-GEO integrated network traffic con-} trol

step 1. Get the traffic flow of each LEO-GEO link through above steps and calculate the correction of discharge coefficient in MLSN $U_{g}$;

step 2. Calculate the Hurst parameter, initialize the constant coefficient $P$ and the attenuation factor $K_{g}$;

step 3. Calculate the input flow of each LEO within the coverage area of GEO $T_{g}^{\prime}$ according to Eq.20, where $T_{g}$ refers to input flow in the previous time slot:

$$
T_{g}^{\prime}=\left\{\begin{array}{l}
U_{g} \times T_{g}, U_{g} \geq 1 \\
T_{g} \times \frac{1}{1+K_{g}}, 1>U_{g}>0
\end{array}\right.
$$

step 4. Modify the input flow coming from each LEO to GEO by Eq.21:

$$
T=\min \left(T_{g}^{\prime}, T_{o}-\left(T_{i(A)}{ }^{\prime}+T_{i(B)}{ }^{\prime}\right)\right)
$$

The above is the calculation process of TCCR. The first stage is divided into five steps, and the second stage is divided into four steps. In chapter 4, we will present the tests and results of the TCCR compared with two existing methods.

\section{SIMULATIONS AND ANALYSIS}

To validate the performance of the proposed scheme, we conducted extensive computer simulations by using OPENT. Here, we discuss the effectiveness of the proposed traffic control scheme based on chain reaction model compared with the Dijkstra shortest path (DSP) routing and the LBQP algorithm proposed in literature [2] and then review the results of our simulation experiments.

\section{A. Network Configuration and Communication Scenarios}

We use a two-layered satellite networks composing of LEO and GEO satellites as depicted in Fig. 1. Three equally positioned GEO satellites construct a ring constellation as the upper layer, while the Iridium constellation consisting of 66 LEO satellites is assumed as the lower layer. The bandwidth and other parameters of the satellite system are set up according to Table 1 .

TABLE I.

PARAMETERS IN THE PROPOSED SCHEME

\begin{tabular}{|c|c|}
\hline LEO & 66 \\
\hline GEO & 3 \\
\hline Packet Size & $1 \mathrm{~KB}$ \\
\hline On-period & $0.4 \mathrm{~s}$ \\
\hline Off-period & $0.8 \mathrm{~s}$ \\
\hline ISL in GEO layer & $25 \mathrm{Mbps}$ \\
\hline ISL in LEO layer & $2.5 \mathrm{Mbps}$ \\
\hline ILL & $25 \mathrm{Mbps}$ \\
\hline GSL & $5 \mathrm{Mbps}$ \\
\hline
\end{tabular}

\section{B. Performance and Discussion}

In performance evaluation, packet drops, throughput, and end-to-end delays are used as performance metrics.

Fig. 7 presents the packet drop rates of the three different mechanisms. The red and blue area graph represents bitrate per flow corresponds to right-Y axes. As shown in Fig.7, the proposed scheme has much better performance than DSP and LBQP proposed in literature [2] due to its improved threshold calculation method. And a more reasonable traffic flow forwarding strategy is obtained through TCCR by introducing the Hurst factor and other parameters. Moreover, the proposed scheme succeeds in coping with network traffic burst compared with LBQP. 
When traffic flow increases in a short time, TCCR will limit the input flow of each satellite by calculating the discharge coefficient and attenuation factor, to ensure that the LEO traffic data remains a relatively stable value.

Fig. 8 indicates the PDR versus average bitrate of various algorithms. In the proposed scheme employing an intelligent forwarding mechanism, the packet drop rate is successfully decreased by $34.14 \%$ when the average bitrate per flow over $250 \mathrm{Kbps}$ compared with LBQP.

Fig.9 presents the throughput of the three different mechanisms. The red and blue area graph represents bitrate per flow corresponds to right-Y axes. As shown in the simulation result, the throughput of TCCR and LBQP is not sensitive to the change of the instantaneous bitrate per flow. This is because the TCCR and LBQP both adopt the corresponding flow control mechanism. In TCCR, the traffic flow sending to GEO in stage 1 will be modified by a corrective feedback operation in stage 2. Therefore, compared with LBQP, TCCR can more efficient use of idle LEOs to forwarding and processing traffic flow of satellite network, so as to make the system to achieve higher throughput performance. Figure 10 shows the total throughput of the three kinds of algorithms versus average bitrate.

Fig.11 indicates the end-to-end delay versus average bitrate of various algorithms. LBQP and TCCR spend more time in controlling and allocating resource due to its high complexity which leads to a bad performance compared with DSP. But the PDR and throughput performance of DSP is too poor so the delay of DSP is only for reference. Next we mainly analyze the delay performance of LBQP and TCCR.

As shown in Fig. 11, TCCR has relatively longer delays at the low bitrates. The delay increases as bitrates increasing, but the increased degree of TCCR decreases while LBQP is exactly the opposite. This is because the fact that what LBQP focus on is the actual flow numerical calculation, and the computing time is prolonged remarkably with the bitrates increasing. Instead, what TCCR focus on is the variation characteristic of parameters which can be obtained by the cross-correlation between different satellites. Therefore, the computational complexity will not increase with the increasing of the bitrate making the proposed scheme succeeds in decreasing the delay compared with LBQP. Fig. 12 presents the delay of the three different mechanisms when bitrate over $300 \mathrm{Kbps}$. The red and blue area graph represents bitrate per flow corresponds to right-Y axes. The proposed scheme is able to decrease the delay as a result of the reduction of parameters calculation and threshold setting when network congestion occurs in a high bitrate.

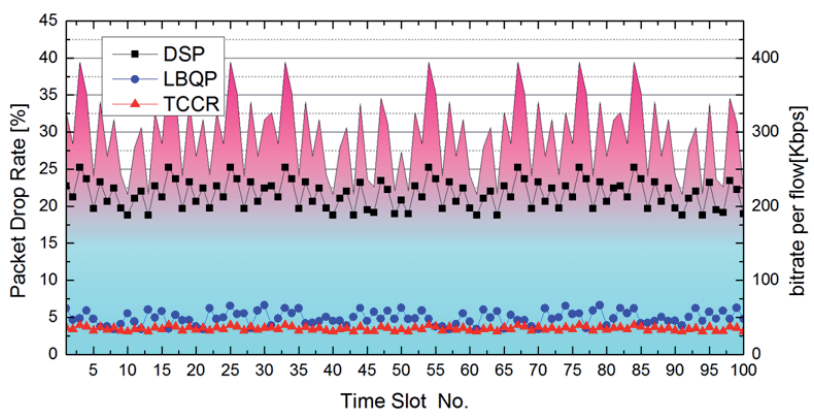

Figure 7. PDR versus bitrate of various algorithms

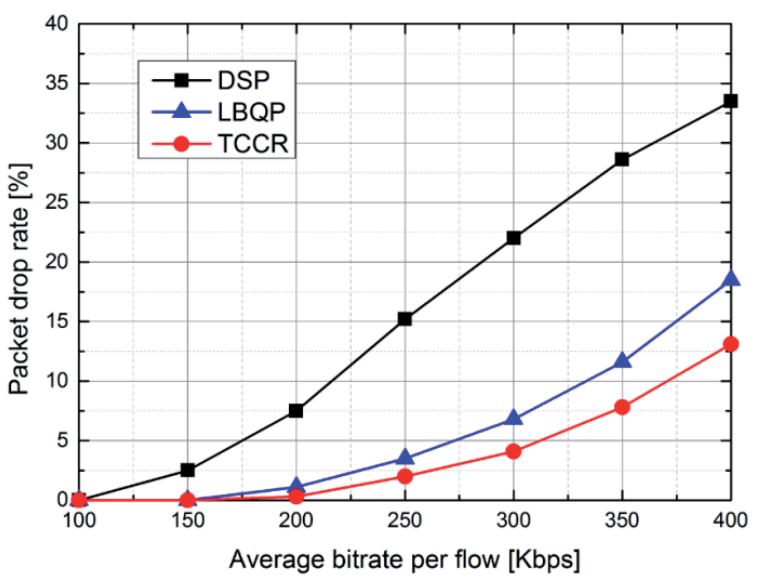

Figure 8. PDR versus average bitrate of various algorithms

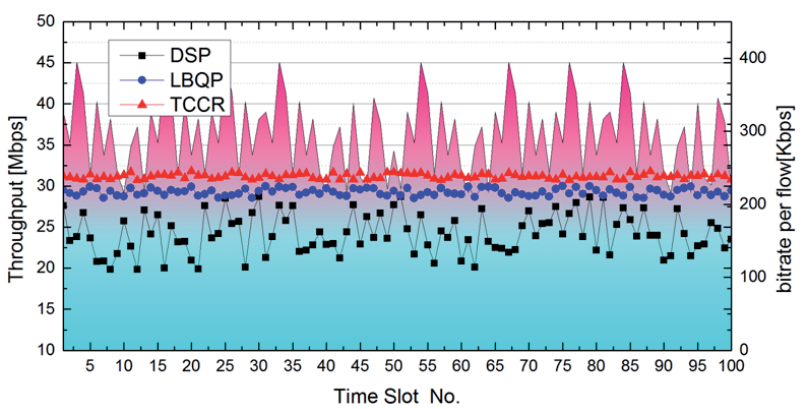

Figure 9. Total throughout versus bitrate of various algorithms

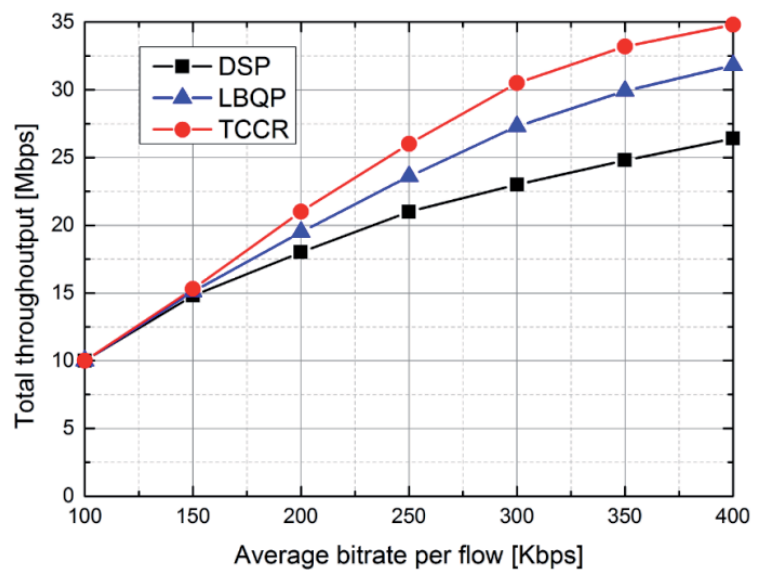

Figure 10. Total throughout versus average bitrate of various algorithms

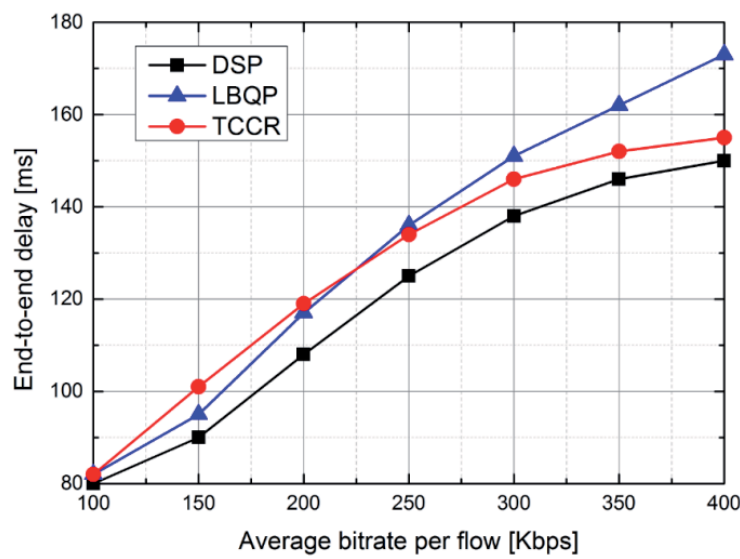

Figure 11. Delay versus average bitrate of various algorithms 


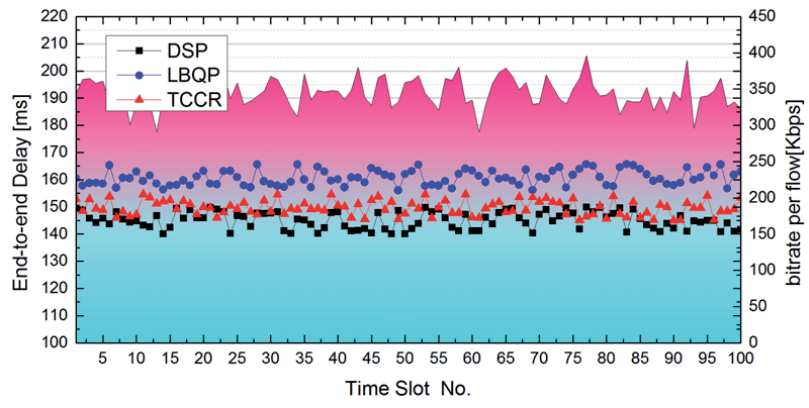

Figure 12. Delay versus bitrate over $300 \mathrm{Kbps}$ of various algorithms

\section{CONCLUSION}

With the development of the wireless communication technology, the demand for broadband communication services has increased rapidly. In order to handle the shortage existing in the previous traffic control scheme, a novel traffic control method based on chain reaction model (TCCR) is presented in this paper to realize dynamic data transmission and control for MLSN. The optimal flow distribution scheme will be given by analyzing the network transmission information in this algorithm. Simulation by OPENT is done which proves that TCCR can obviously improve the network total throughput by $7.24 \%$, shorten link transmission delay by $6.3 \mathrm{~ms}$, and reduce packet loss rate by $34.14 \%$ compared with LBQP when bitrate is more than $300 \mathrm{Kbps}$. Through extensive computer simulations, the superior performance of the proposed scheme is verified.

\section{REFERENCES}

[1] Xiaoyou Yu; Yu Yang; Jinjun Ding, "Satellite network design method applicable to orbit determination and communication for GNSS," Software Engineering and Service Science (ICSESS), 2013 4th IEEE International Conference on , vol., no., pp.886,889, 23-25 May 2013

[2] Nishiyama, H.; Kudoh, D.; Kato, N.; Kadowaki, N., "Load Balancing and QoS Provisioning Based on Congestion Prediction for GEO/LEO Hybrid Satellite Networks," Proceedings of the IEEE , vol.99, no.11, pp.1998,2007, Nov. 2011

[3] Cen Qian; Sihai Zhang; Wuyang Zhou, "Traffic-based dynamic beam coverage adjustment in satellite mobile communication," Wireless Communications and Signal Processing (WCSP), 2014 Sixth International Conference on, vol., no., pp.1,6, 23-25 Oct. 2014 http://dx.doi.org/10.1109/wcsp.2014.6992014

[4] Kawamoto, Y.; Nishiyama, H.; Kato, N.; Yoshimura, N.; Kadowaki, N., "A delay-based traffic distribution technique for Mul-
ti-Layered Satellite Networks," Wireless Communications and Networking Conference (WCNC), 2012 IEEE , vol., no., pp.2401,2405, 1-4 April 2012

[5] Petropoulou, P.; Michailidis, E.T.; Panagopoulos, A.D.; Kanatas, A.G., "Radio Propagation Channel Measurements for MultiAntenna Satellite Communication Systems: A Survey," Antennas and Propagation Magazine, IEEE, vol.56, no.6, pp.102,122, Dec. 2014

[6] Xufeng Peng; Zhangwei Chen; He Mao, "Adaptive fuzzy PID control for polymerase chain reactions," Biomedical Engineering and Informatics (BMEI), 2013 6th International Conference on , vol., no., pp.337,341, 16-18 Dec. 2013 http://dx.doi.org/10.1109/bmei.2013.6746959

[7] Wei-Chang Yeh, "The Extension of Universal Generating Function Method to Search for All One-to-Many d -Minimal Paths of Acyclic Multi-State-Arc Flow-Conservation Networks," Reliability, IEEE Transactions on , vol.57, no.1, pp.94,102, March 2008

[8] Schweitzer, J.S.; Ziehl, W., "Temperature Dependence of NaI(Tl) Decay Constant," Nuclear Science, IEEE Transactions on , vol.30, no.1, pp.380,382, Feb. 1983

[9] Dongchen Li; Penglang Shui, "Floating small target detection in sea clutter via normalised Hurst exponent," Electronics Letters , vol.50, no.17, pp.1240,1242, Aug. 142014

[10] Li Li; Zhiheng Li; Yi Zhang; Yudong Chen, "A Mixed-Fractal Traffic Flow Model Whose Hurst Exponent Appears Crossover," Computational Sciences and Optimization (CSO), 2012 Fifth International Joint Conference on , vol., no., pp.443,447, 23-26 June 2012 http://dx.doi.org/10.1109/cso.2012.103

\section{AUTHORS}

Ning Li, female, Ph.D., associate professor at the School of Electronic Engineering, Beijing University of Posts and Telecommunications. Research Interests: Information Security, Satellite Communications.

Pengfei Wang, male, graduate student at the school of Electronic Engineering, Beijing University of posts and telecommunications. Research Interest: Satellite Communications, Traffic Control.

Zhongliang Deng, male, Ph.D., professor at the School of Electronic Engineering, Beijing University of Posts and Telecommunications. Research Interests: Satellite Navigation and Communications.

Ke Han, male, Ph.d., lecturer at the School of Electronic Engineering,Beijing University of Posts and Telecommunications. Research Interests: IC design, Satellite Communications.

Submitted, 09 March 2015. Published as resubmitted by the authors on 09 April 2015. 